\title{
Blood Screening
}

National Cancer Institute

\section{Source}

National Cancer Institute. Blood Screening. NCI Thesaurus. Code C15960.

Examination of the blood from a group of usually asymptomatic individuals to detect those with a high probability of having a given disease, typically by means of inexpensive diagnostic tests. 